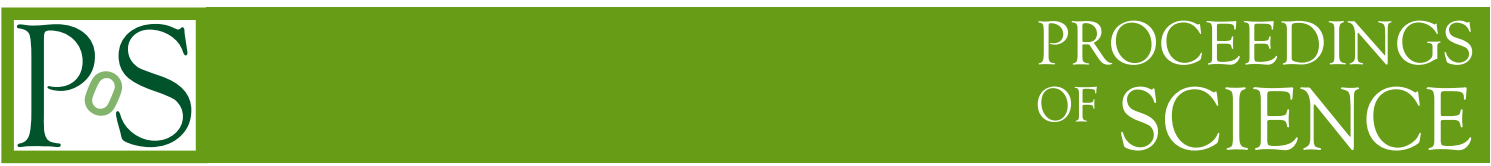

\title{
The new "Gauge Connection" at NERSC
}

\author{
Massimo Di Pierro* \\ School of Computing - DePaul University - Chicago \\ E-mail: mdipierro@cs.depaul.edu \\ James Hetrick \\ University of the Pacific \\ E-mail: jhetrick@pacific.edu
}

\section{Shreyas Cholia}

Lawrence Berkely Laboratory

E-mail: scholiaelbl.gov

\section{James Simone}

Fermi National Accelerator Laboratory

E-mail: simonedfnal.gov

\section{Carleton DeTar}

University of Utah

E-mail: detaraphysics.utah.edu

In this paper we present a new web interface (NERSC3) for the Gauge Connection, one of the largest repositories of lattice gauge configurations in the US. The Gauge Connection is hosted at the National Energy Research Scientific Computing Center (NERSC), and stores more than 16TB of data using the High Performance Storage System (HPSS). The new interface allows users to search, download, and view statistics on lattice QCD ensembles hosted at NERSC. It also aggregates metadata from other major lattice archives (ILDG), and allows one to search the metadata in a single place. Additionally, users can tag, annotate, and bookmark the ensembles (http://qcd.nersc.gov).

31st International Symposium on Lattice Field Theory - LATTICE 2013

July 29 - August 3, 2013

Mainz, Germany

\footnotetext{
*Speaker.
} 


\section{Introduction}

The Gauge Connection is one of the most popular repositories of lattice QCD ensembles. It has been operated by the National Energy Research Scientific Computing Center [1] (NERSC) since 1998, and currently stores over 16 Terabytes of data. The popularity of the Gauge Connection can be attributed to the amount of data it stores, and its easy-to-use web interface which lowers the barrier of entry for lattice QCD researchers.

At the core of the Gauge Connection architecture is the High Performance Storage System [2] (HPSS) data archive. The HPSS system provides a tape archival storage library for the QCD data. The content of the archive is exposed using an FTP interface in conjunction with a CGI-based HTTP download service.

The original web interface consisted of static HTML files linked against this CGI service that would pull data from the HPSS archive. In 2011 the web interface underwent a major revision and the static pages were replaced by dynamically generated pages. The content of the pages comprised of free text mined from the original HTML files, which was made editable in a wiki-like interface. The pages were augmented with metadata obtained from the file catalog itself and stored in a database. The new interface allowed users to search the data more easily, comment on the data, and download ensembles in batch.

In this paper we present a new version of this service which we will refer to as NERSC3. Apart from user interface changes, the main functional change is an expanded search capability which allows users to search both local and remote repositories of ensembles.

Lattice QCD physicists formed the International Lattice Data Grid (ILDG) [3] collaboration in 2000, and developed protocols and infrastructures to annotate and share lattice QCD ensembles. They created a file format to store gauge ensembles along with standardized metadata, and a network of ILDG metadata catalogs and file catalogs. This enabled searching against the ensembles and their metadata, and the ability to download the files.

The ILDG infrastructure is powerful but compared to the NERSC archive, it presents two major barriers of entry: 1) By design, the metadata catalogs do not inter-operate but provide a common search API; therefore search is decentralized. 2) The file catalogs are built on top of GridFTP [5] and can only be accessed using ILDG-tools, and only after obtaining a valid X509 grid certificate.

In our latest revision of the NERSC archive we have been addressing the first issue. The new system periodically connects to all of the ILDG metadata catalogs and copies the ensemble metadata to a local database. This allows a visitor of the site to search for both local ensembles and remote ILDG ensembles in one place. While the local data can be retrieved directly from the web site or via the provided batch download script, remote data is only referenced, and the user must use the ILDG tools to query the remote file catalog and use GridFTP to download the data.

Moreover, conversion to the ILDG data format has been slow: the bulk of the data is stored in other formats, e.g. NERSC or MILC while only a fraction is stored in ILDG format. Additionally, one of the key improvements to NERSC3 includes the ability to convert file formats post-download.

We have designed the new website to provide additional capabilities beyond data access, including wiki capabilities to annotate the data, link external work derived from the data (derived 


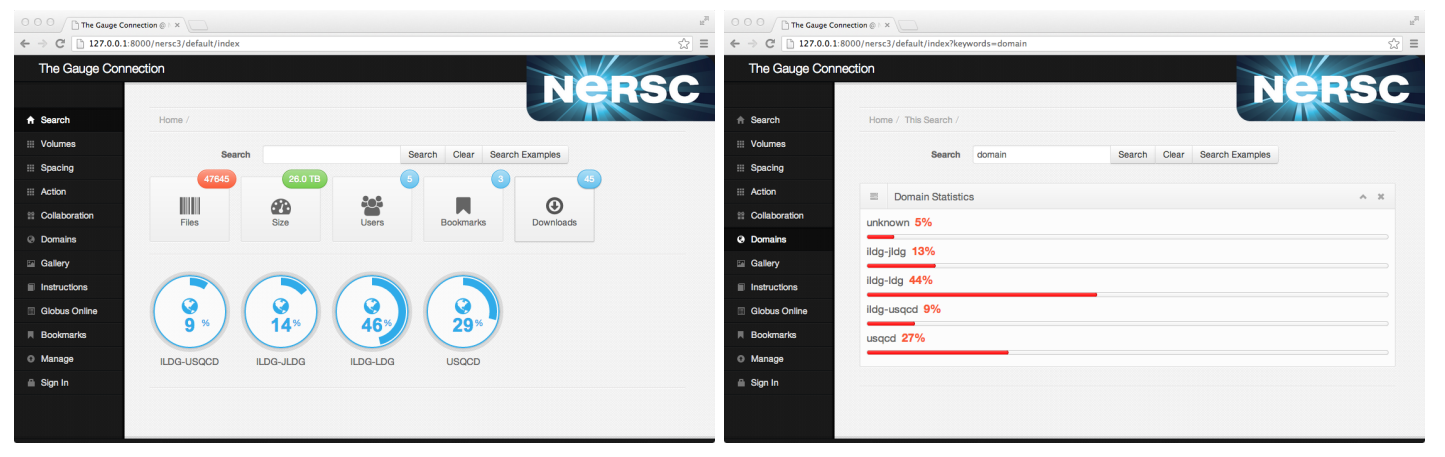

Figure 1: The main web page for qcd. nersc. gov allows browsing and searching for gauge ensembles (left) and visualizing statistical data about the ensembles (right).

data, software, tutorials, and publications), and bookmark interesting data. The new system keeps usage statistics for analytics purposes.

Figure 1 and figure 2 show some screenshots from NERSC3.

\section{Architecture Overview}

The Gauge Connection is hosted at NERSC on the High Performance Storage System (HPSS). HPSS is a hierarchical tape storage system designed to store multiple petabytes which be accessed using a custom command line client (HSI), a C API, an FTP interface, a parallel FTP interface, as well as a GridFTP interface. The latter can also be accessed using Globus Online, a service that enables scheduled data transfers between GridFTP endpoints.

NERSC3 periodically performs directory dumps of the HPSS tapes and reproduces the folder structure in the database. NERSC3 has no direct access to the files because staging them from the tape archive is expensive. Yet it can infer some information about the content of the files from the names of the folders, the names of the files, and their sizes. Most of the files stored at NERSC are produced by the MILC collaboration which is one of the major sources of public gauge ensembles in the world. The MILC collaboration adopts a practical naming convention for the file names which include information about the lattice volume, the kappa value, and the quark masses (for dynamical ensembles) and a progressive configuration counter. NERSC 3 can link this information to additional metadata obtained from other sources which is inserted in the system manually e.g. links to papers describing the algorithm used to generate each ensemble.

The NERSC3 database stores information about two types of ensembles: the ensembles stored at NERSC described above and ILDG ensembles stored at other locations.

The ILDG infrastructure consists of the following components:

- A description of metadata which accompanies each ensemble. This includes name of the group/collaboration, description of the action and algorithms used to generate it, simulation parameters (lattice volume, lattice spacing, dynamical quark masses) and links to relevant papers. The information is encoded in a custom XML-based schema. 
- A file format for storing data along with metadata. ILDG uses a custom encoding protocol similar to TAR, called LIME.

- A metadata catalog service which exposes SOAP services to query the catalog for local ensembles, their metadata, and their file content.

- A file catalog service which exposes additional SOAP services, protected by WS-Security, to convert logical filenames into actual URLs for downloading the files.

ILDG is comprised of 5 realms: CSSM (Australia), LDG (Europe), JLQCD (Japan), UKQCD (UK), USQCD (US). Each realm runs at least one metadata catalog service and one file catalog service.

In ILDG each ensemble is identified by a Unique Resource Identifier (URI) starting with the mc: prefix. Each logical file name is identified by a URI starting with the $l \mathrm{fn}$ : prefix. The actual file names are not URIs but they are URLs. They usually have one of the following prefixes: http:, ftp:, https:, ftps:, and srm:. srm: is a GridFTP based web service protocol which operates over HTTP but is designed to act as a referrer for multiple endpoints and effectively load-balances transfers over multiple nodes, allowing transfers to scale. It is very similar in scope to the BitTorrent protocol, i.e. it splits data transfers over multiple connections. The SRM protocol is supported by the $d$ Cache [7] tape storage system used at Fermilab.

The metadata catalog services are public. NERSC3 periodically connects to these services and downloads a complete list of available ensembles, their metadata, and their logical filenames. This information is stored in a local database. In this way, when using the NERSC3 web interface one can search both the local ensembles and all the ensembles made available via ILDG.

The ILDG file catalogs are not public. This means that the conversion between logical filename to actual URLs cannot be performed without a valid GridFTP certificate, and each realm has its own policies and procedures for assigning certificates. Accessing the actual URLs also requires a certificate. Thus, while NERSC3 allows for searching of remote data and can point the user to a specific remote ensemble, it does not help the user with accessing the data itself.

Only the data stored at NERSC is truly public in the sense that it can be accessed by anybody without explicit permission from an ILDG certificate authority.

Figure 3 shows an overview of the architecture described here.

In NERSC3 tags are associated with ensembles, rather than individual files. Each tag is divided into two parts separated by a colon (:). e.g. volume: $24 \wedge 3 \times 48$. The first part of the tag (in this example volume) is a key, while the second part (in this example $24 \wedge 3 \times 48$ ) is a value. Tags are all optional and key names are not dictated by the system but chosen by the users. Some keys may not have a corresponding value. More examples of tags include beta:2.1, collaboration:milc, and flavor: $2+1$. When clicking on a tag, the user is presented with a list of ensembles sharing the same tag.

Users can search by key (any key) and they are presented with a list of all possible values for that key along with statistical information. They can search by tag (key:value) and they are presented with a list of ensembles with the corresponding tag. They can search for multiple tags and they are presented with a list of ensembles that have all the requested tags. Here are some examples of search strings: 


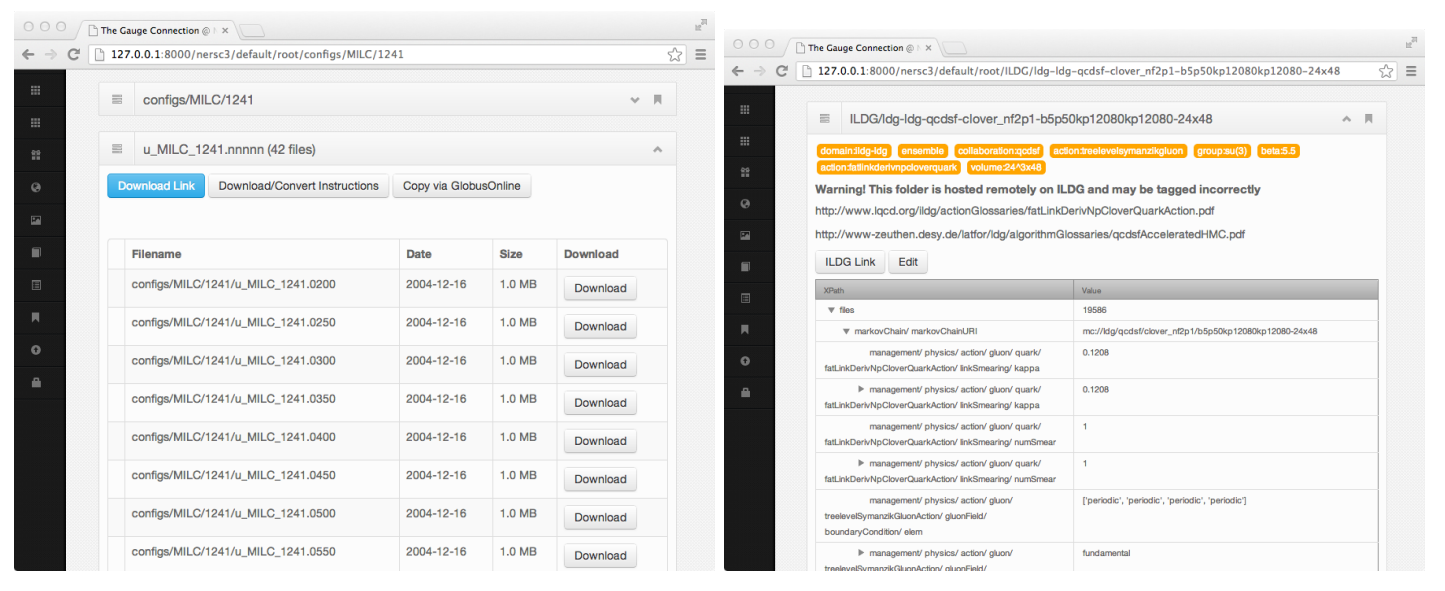

Figure 2: For each local ensemble, the NERSC3 interface can list the ensemble files (left). For remote ensembles, the NERSC3 interface can list the ILDG metadata and the FLN URI.

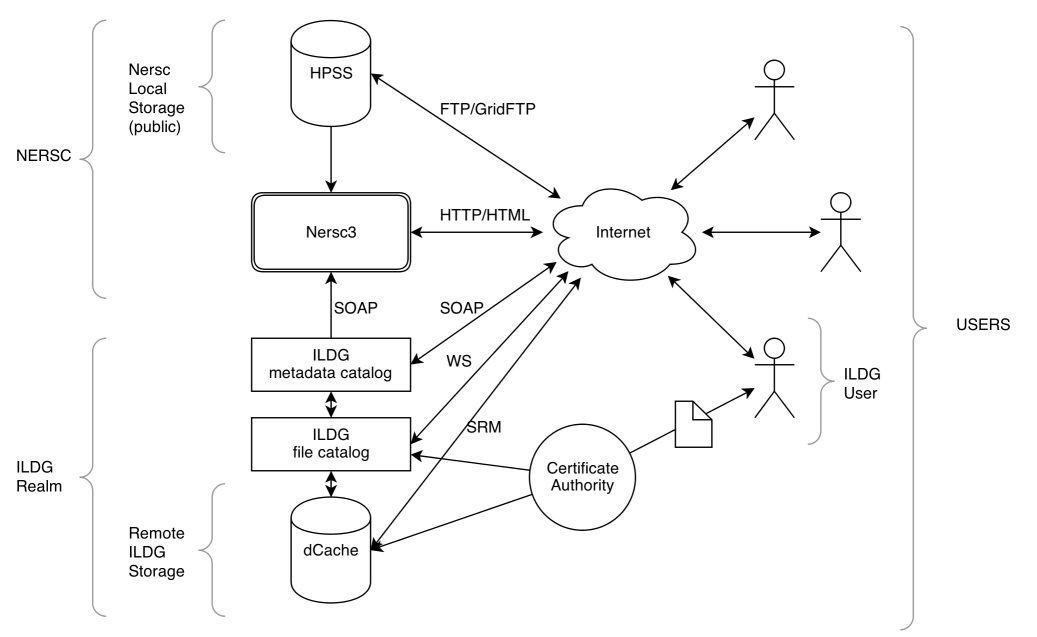

Figure 3: Overview of the NERSC3 and ILDG architecture.

1 volume

2 volume: $12^{\wedge} 3 \times 12$

3 volume is $12^{\wedge} 3 \times 12$

4 volume is $12^{\wedge} 3 \times 12$ and domain is usqcd and collaboration is milc

In this case the domain "usqcd" refers to the set of ensembles that are hosted at NERSC as opposed to the ILDG ensembles.

The search string understands the "is" and "and" english words. A search string can also be the name of an ensemble.

Figure 4 shows the database architecture of the NERSC3 application. It includes tables to store users, groups, memberships and permissions. We implement role based access control for administrators. It includes a table catalog_folder (which represents an ensemble) and a table catalog_file which stores links to individual files (the files themselves are in NERSC HPSS or ILDG). ildg metedata, accesslogs, bookmarks, and tags reference the catalog_folder 


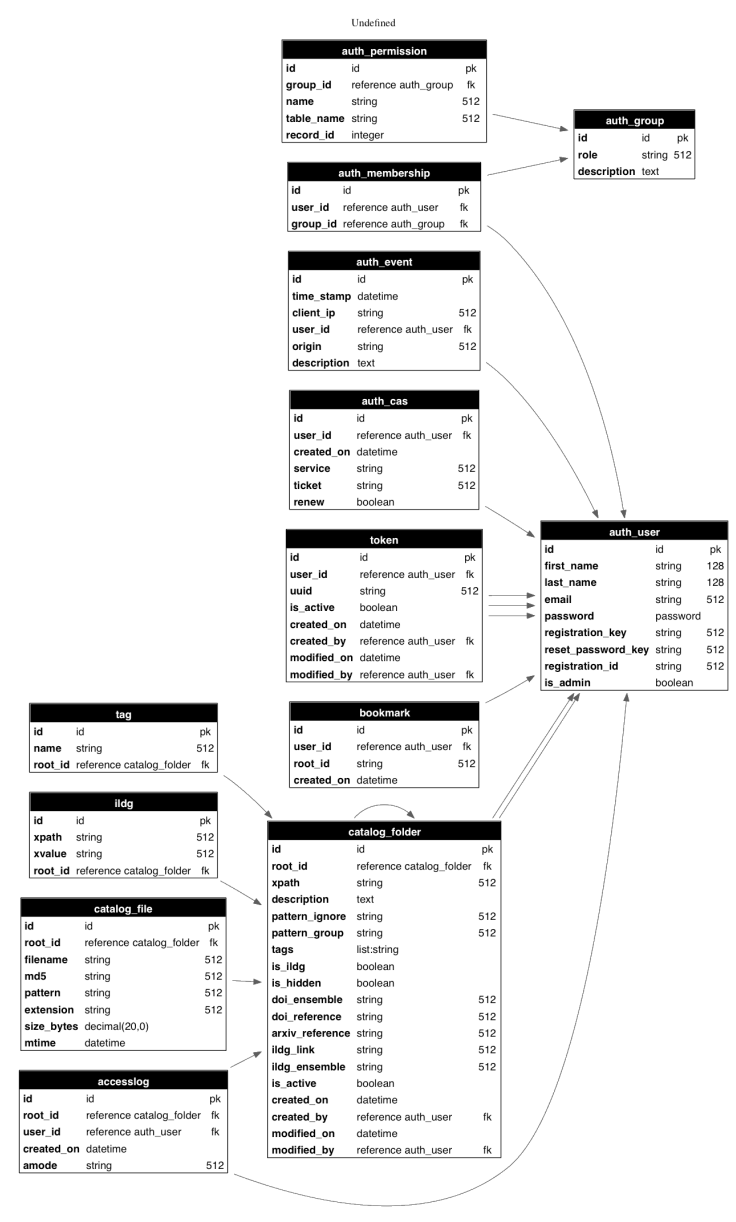

Figure 4: Overview of the table structure of NERSC3. The table catalog_folder represents ensembles and the table catalog_file represents files in the ensembles.

table. Logged in users are also issued tokens which are used to authenticate users for batch downloads. NERSC3 is based on the web2py [8] framework.

\section{The batch download script}

NERSC3 allows users to download local ensemble files individually or in batch. Individual downloads can be performed directly from the web pages. For batch downloads, the web page of each local ensemble provides a link to a JSON file containing a list of files in the ensemble. A user does not need to open this file, but only needs to copy its URL, and can then use the qcdutils_get utility from qcdutils [9] to download all files referenced by the link. For example if the link is http://qcd.nersc.gov/nersc/api/files/demo then the user would download the files with the following command:

1 > qcdutils_get.py http://qcd.nersc.gov/nersc/api/files/demo

2 http://qcd.nersc.org/nersc/api/files/demo

3 target folder: demo

4 total files to download: 1 
5 downloading demo.nersc

6 demo.nersc 100\% |\#\#\#\#\#\#\#\#\#\#\#\#\#\# Time: 00:00:00 $654.52 \mathrm{~K} / \mathrm{s}$

7 completed download: 1/1

The qcdutils_get script will download the demo file, read its contents, and sequentially download all the files listed in there. It shows download progress and keeps a record of completed downloads. If restarted, it resumes from the last completed download.

It also has the ability to convert ensembles to different formats. For example, here we ask to convert the most recent downloads to single precision IDLG format.

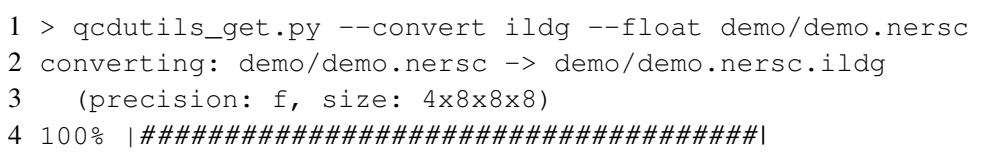

It keeps a log of all completed operations to avoid duplication of work. One can query qcdutils for a $\log$ of the completed tasks. For example:

1 > qcdutils_get.py demo/qcdutils.catalog.db

2 demo.nersc created on 2011-06-17T13:42:30.876812

3 [14e7cf9106bfcc16388aeac285ccdad9]

We refer to the qcdutils [9] code and manual for details.

\section{Conclusions and Outlook}

In this paper we have presented a new interface to the Lattice QCD Gauge Connection at NERSC. The purpose of the new interface is to lower the barrier of entry for lattice QCD researchers, and to allow users of the archive to search both local and remote ensembles of lattice gauge configurations. It also allows scientists to access metadata about the ensembles, and to contribute to the metadata by editing wiki entries associated to them.

\section{Acknowledgments}

This research used resources of the National Energy Research Scientific Computing Center, which is supported by the Office of Science of the U.S. Department of Energy under Contract No. DE-AC02-05CH11231.

\section{References}

[1] http://qcd.nersc.gov

[2] http://www.hpss-collaboration.com

[3] Maynard, Chris M., arXiv preprint arXiv:1001.5207 (2010).

[4] Beckett, Mark G. et al., Comp.Phys.Comm. 182 (2011) pp.1208-1214

[5] Yildirim, Esma et al.,High Performance Computing, Networking, Storage and Analysis (SCC), 2012 SC Companion:. IEEE, 2012.

[6] Di Pierro, Massimo, Nucl. Phys. B-Proceedings Supplements 106 (2002): 1034-1036.

[7] Agarwal, A., et al. Journal of Physics: Conference Series. Vol. 219. No. 7. IOP Publishing, 2010.

[8] Di Pierro, Massimo, Computing in Science and Engineering 13.2 (2011): 64-69.

[9] Di Pierro, Massimo, arXiv preprint arXiv:1202.4813 (2012). 